\title{
Control of Untethered Magnetically Actuated Tools using a Rotating Permanent Magnet in any Position
}

\author{
Arthur W. Mahoney, Daniel L. Cowan, Katie M. Miller, and Jake J. Abbott
}

\begin{abstract}
It has been shown that when a magnetic dipole, such as a permanent magnet, is rotated around a fixed axis such that the dipole is perpendicular to the axis of rotation, the magnetic field vector at every point in space also rotates around a fixed axis. In this paper, we reformulate this phenomenon using linear algebraic techniques, which enables us to find the necessary dipole rotation axis to make the magnetic field at any desired point in space rotate about any desired axis. To date, untethered magnetically actuated tools (e.g., capsule endoscopes, rolling spheres, and helical-propeller microswimmers) controlled with a single rotating permanent magnet have been constrained to operate in positions where the rotating field behavior is simple and easy to visualize. We experimentally demonstrate that the results of this paper can be used to control a variety of untethered, rotating magnetic devices in any position even while the rotating permanent magnet follows trajectories independent of the devices themselves. This method constitutes a substantial step toward making a great deal of prior laboratory research regarding rotating magnetic microrobots and capsule endoscopes clinically feasible.
\end{abstract}

\section{INTRODUCTION}

Untethered magnetic devices, such as magnetic microrobots [1] and magnetically actuated capsule endoscopes [2], have become an active area of research because of their potential impact to minimally invasive medicine. These devices typically consist of a rigidly attached magnetic body on which magnetic forces and torques are applied by an external field. Some approaches to actuation utilize magnetic forces for pulling [3], [4], while others apply torque generated by rotating magnetic fields to roll on a surface [5]-[7], swim through a fluid or crawl through a lumen via helical propulsion [8]-[12], or screw through soft tissue [13]. Because these devices can be viewed as simple end-effectors of a larger robotic system, and they may range in size from the microscale to the mesoscale, we refer to them herein as magnetically actuated tools (MATs) without any implied size.

MATs are typically actuated using applied magnetic fields produced by electromagnets. Such systems often take the form of orthogonal arrangements of Helmholtz coils, which generate uniform fields, and Maxwell coils, which generate uniform field gradients. Because electromagnetic systems are expensive to scale to the size required for clinical use, researchers are considering actuation using a single permanent magnet [5]-[9]. Although likely less expensive, actuation with permanent magnets is significantly more complex due

This material is based upon work supported by the National Science Foundation under Grant Nos. 0952718 and 0654414

A. W. Mahoney and K. M. Miller are with the School of Computing, and D. L. Cowan and J. J. Abbott are with the Department of Mechanical Engineering, University of Utah, Salt Lake City, UT, 84112 USA. art.mahoney@utah.edu

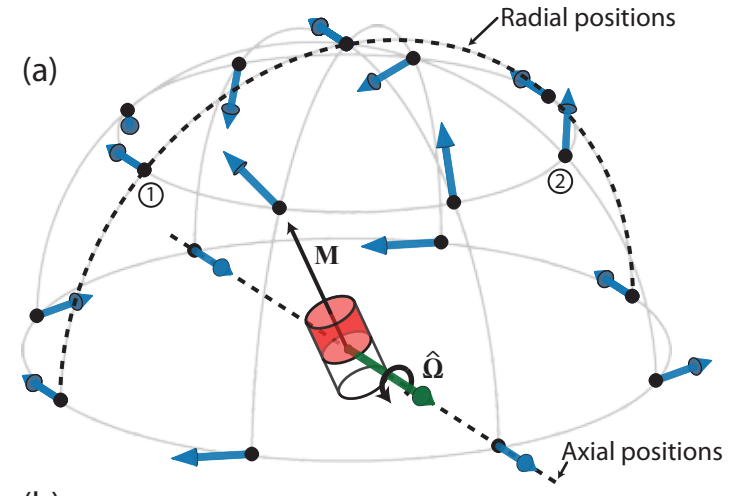

(b)

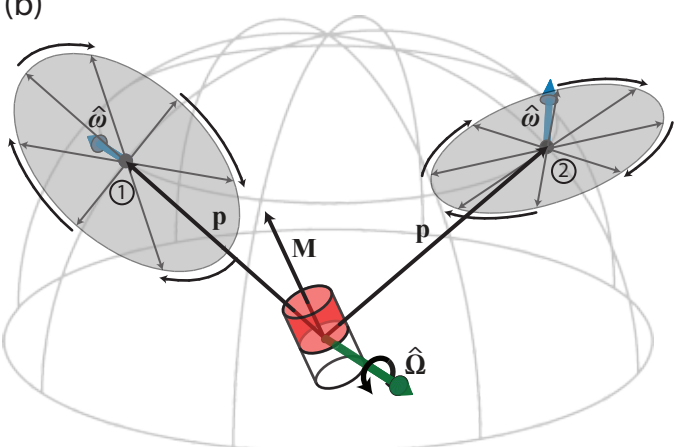

Fig. 1. When a dipole magnet with moment $\mathbf{M}$ rotates around the axis $\hat{\Omega}$ with $\mathbf{M}$ perpendicular to $\hat{\Omega}$, the field vector at any given position rotates around, and is perpendicular to, a constant axis $\hat{\omega}$. The axis $\hat{\omega}$ at various positions are illustrated with large blue arrows in (a). Any position on the $\hat{\mathbf{\Omega}}$ axis is denoted to be in ax axial position and any position in the plane spanned by the rotating $\mathbf{M}$ is a radial position. Representations of the field behavior at locations 1 and 2 are detailed in (b). The magnetic field $\mathbf{H}$, at locations 1 and 2, is illustrated by gray arrows rotating around $\hat{\boldsymbol{\omega}}$.

to nonuniformity of the generated magnetic fields. In the case of MAT locomotion using rotating applied fields produced by a single rotating permanent magnet (RPM), the RPM is typically rotated around an axis $\hat{\Omega}$ such that the RPM's dipole moment $\mathbf{M}$ is always perpendicular to $\hat{\Omega}$ as depicted in Fig. 1. To date, MATs have been exclusively operated in radial or axial positions (relative to the RPM) where the applied field rotates around an axis parallel to $\hat{\Omega}$ (see Fig. 1(a)) [5]-[9]. The axial and radial positions have been historically favored for actuation due to their simplicity: the rotation axis $\hat{\omega}$ of the applied magnetic field in both positions lies parallel to the RPM axis of rotation, making it easy to visualize and characterize the coupling between the RPM and the MAT. Requiring the MAT to be exclusively operated in these two positions, however, significantly constrains the physical placement of the RPM. This may become problematic and 
limit usefulness in a clinical setting since the RPM must move during actuation to avoid collisions with the patient and other obstacles, and to reposition for better control authority.

It has been shown that when a dipole (e.g., RPM) rotates such that its moment is always perpendicular to the axis of rotation, the generated field $\mathbf{H}$ at any position in space rotates around, and is perpendicular to, a constant axis (Fig. 1(a)) with field magnitude described by an ellipse (Fig. 1(b)) [14]. We begin by reformulating this phenomenon in a manner that readily enables the solution of the inverse problem: finding the necessary RPM rotation axis $\hat{\Omega}$ given a desired applied field rotation axis $\hat{\omega}$, for any RPM position relative to the MAT. The results enable a rotating magnetic field to be produced around an arbitrary axis $\hat{\omega}$ in space using a single RPM in any position. We demonstrate the theory presented in this paper by driving a threaded capsule and rolling a spherical MAT through a lumen using an RPM positioned in space by a 6-DOF robotic manipulator. Eliminating the need to operate rotating MATs solely in the axial or radial positions is a significant step toward making rotating MATs actuated by a single RPM clinically realistic.

\section{Controlling Local Field Rotation}

All vectors described in this paper are expressed in a common, static coordinate frame, and the "hat" symbol denotes a vector normalized to unit length. Let the RPM's dipole moment $\mathbf{M}$ rotate around the axis $\hat{\Omega}$ such that $\mathbf{M}$ is always perpendicular to $\hat{\Omega}$, and the direction of rotation is found using a right-hand rule. Assuming the magnetic field generated by the RPM can be accurately modeled with the point-dipole model [15], the magnetic field $\mathbf{H}$ at a position $\mathbf{p}$ relative to the center of the RPM is

$$
\mathbf{H}=\frac{1}{4 \pi|\mathbf{p}|^{3}}\left(3 \hat{\mathbf{p}} \hat{\mathbf{p}}^{T}-\mathbb{I}\right) \mathbf{M}=\frac{1}{4 \pi|\mathbf{p}|^{3}} \mathbb{H} \mathbf{M},
$$

where $\mathbb{I}$ is the identity matrix and $\hat{\mathbf{p}}$ is the unit vector in the direction of $\mathbf{p}$. The matrix $\mathbb{H}$ is symmetric and is purely a function of $\hat{\mathbf{p}}$. Let the vector $\hat{\boldsymbol{\omega}}$ be the desired rotation axis of the local magnetic field at the position $\mathbf{p}$, where $\hat{\omega}$ is normal to the plane containing $\mathbf{H}$, such that $\mathbf{H}^{T} \hat{\boldsymbol{\omega}}=0$ for all $\mathbf{M}$. Replacing $\mathbf{H}$ with (1) in this expression and utilizing the symmetry of $\mathbb{H}$ yields

$$
\mathbf{H}^{T} \hat{\boldsymbol{\omega}}=\frac{1}{4 \pi|\mathbf{p}|^{3}} \mathbf{M}^{T} \mathbb{H} \hat{\boldsymbol{\omega}}=0 .
$$

Because $\mathbb{H} \hat{\boldsymbol{\omega}}$ is constant and $\mathbf{M}^{T} \hat{\boldsymbol{\Omega}}=0$, the solution for the necessary dipole axis of rotation $\hat{\Omega}$ where $\hat{\Omega}$ is parallel to $\mathbb{H} \hat{\boldsymbol{\omega}}$ is the only solution that satisfies (2) and is invariant to the rotation of $\mathbf{M}$. Therefore, given $\hat{\boldsymbol{\omega}}$ and $\hat{\mathbf{p}}, \hat{\Omega}$ can be found with

$$
\hat{\Omega}=\widehat{\mathbb{H} \hat{\omega}} \text {. }
$$

Because $\mathbb{H}$ is constructed using the unit vector $\hat{\mathbf{p}}, \mathbb{H}$ only changes with changes in the direction of $\hat{\mathbf{p}}$. This implies that solutions obtained from (3) are invariant to scaling $|\mathbf{p}|$ by moving the RPM nearer to or farther away from the MAT, provided that the direction of $\mathbf{p}$ remains unchanged. Naturally, the magnitude of the field will depend on $|\mathbf{p}|$.
It can be easily verified using (1) that the eigenvectors of $\mathbb{H}$ include $\hat{\mathbf{p}}$ and a 2-dimensional eigenspace consisting of vectors in the plane orthogonal to $\hat{\mathbf{p}}$, with corresponding eigenvalues $\lambda_{1}=2$ and $\lambda_{2,3}=-1$, respectively. Because $\operatorname{det}(\mathbb{H})=\lambda_{1} \lambda_{2} \lambda_{3}=2$ for all $\mathbf{p}, \mathbb{H}$ is always invertible and there exists exactly one dipole rotation axis $\hat{\Omega}$ to generate a desired field rotation about the axis $\hat{\omega}$ for any MAT position p. The forward problem, which gives the local field axis of rotation $\hat{\omega}$ at the position $\mathbf{p}$ given the RPM axis of rotation $\hat{\Omega}$, is found with

$$
\hat{\omega}=\widehat{\mathbb{H}^{-1} \hat{\Omega}} \text {. }
$$

An explicit representation of $\mathbb{H}^{-1}$ can be found using

$$
\mathbb{H}^{2}=\mathbb{H}+2 \mathbb{I},
$$

derived using the definition of $\mathbb{H}$ in (1) and recognizing that $\hat{\mathbf{p}}^{T} \hat{\mathbf{p}}=1$. Multiplying both sides of (5) by $\mathbb{H}^{-1}$ produces the relation $\mathbb{H}=\mathbb{I}+2 \mathbb{H}^{-1}$ and subsequently

$$
\mathbb{H}^{-1}=\frac{1}{2}(\mathbb{H}-\mathbb{I})
$$

requiring no matrix inversion.

The position $\mathbf{p}$ of the MAT relative to the RPM can be measured using a variety of methods such as computer vision, medical imaging, or magnetic localization. With $\mathbf{p}$ and the desired applied field rotation axis $\hat{\omega}$ known, the necessary actuator axis of rotation $\hat{\Omega}$ can be quickly found by (3). As the MAT or the RPM moves and the direction of p changes, (3) must be updated by repacking the matrix $\mathbb{H}$, and a new solution $\hat{\Omega}$ must be produced to maintain the desired applied field rotation axis at $\mathbf{p}$. Theoretically, a desired RPM position trajectory can be established a priori and the necessary $\hat{\Omega}$ can be found during execution given the position of the RPM and the MAT. In practice, however, the RPM position trajectory should adapt to the MAT behavior in order to maintain control authority while simultaneously avoiding hardware collisions or constraints.

\section{Controlling Rotating Magnetically ACTUATED TOOLS}

For rotating MATs, a common failure mode that results in control authority loss occurs when the MAT steps out of synchronization with the rotating local field. The rotation frequency above which the applied magnetic torque is too weak in magnitude to keep the MAT synchronized with the rotating field is referred to as the "step-out" frequency and is denoted by $|\boldsymbol{\omega}|_{\text {so. }}$ If a MAT's angular velocity is proportional to the magnetic torque by a linear damping coefficient $c$ with negligible contribution from inertia, $|\boldsymbol{\omega}|_{\text {so }}$ is the speed where the MAT dipole moment $\mathbf{m}$ lags the applied field by $90^{\circ}$, and requires the maximum possible magnetic torque to maintain synchronization. The maximum magnetic torque is $|\boldsymbol{\tau}|_{\max }=\mu_{0}|\mathbf{m}||\mathbf{H}|$ resulting in the step-out frequency $|\boldsymbol{\omega}|_{\text {so }}=c|\boldsymbol{\tau}|_{\max }$, where $\mu_{0}$ is the permeability of free space.

In rotating uniform magnetic fields, the applied field magnitude and angular velocity remain unchanged regardless of the MAT position. In rotating nonuniform fields produced by an RPM, however, the applied field magnitude and angular 
velocity, at any given MAT position $\mathbf{p}$, depend on the orientation of $\mathbf{M}$ and $\mathbf{p}$. During RPM rotation, the instantaneous field magnitude $|\mathbf{H}|$ fluctuates in an elliptical fashion, and is given by

$$
|\mathbf{H}|=\frac{|\mathbf{M}|}{4 \pi|\mathbf{p}|^{3}} \sqrt{1+3\left(\hat{\mathbf{M}}^{T} \hat{\mathbf{p}}\right)^{2}}
$$

and the minimum and maximum field magnitudes are

$$
\begin{aligned}
|\mathbf{H}|_{\min } & =\frac{|\mathbf{M}|}{4 \pi|\mathbf{p}|^{3}} \\
|\mathbf{H}|_{\max } & =\frac{|\mathbf{M}|}{4 \pi|\mathbf{p}|^{3}} \sqrt{1+3|\tilde{\mathbf{p}}|^{2}},
\end{aligned}
$$

where the vector $\tilde{\mathbf{p}}=\left(\mathbb{I}-\hat{\boldsymbol{\Omega}} \hat{\boldsymbol{\Omega}}^{T}\right) \hat{\mathbf{p}}$ is the projection of $\hat{\mathbf{p}}$ onto the plane perpendicular to $\hat{\Omega}$ (the plane in which $\mathbf{M}$ resides). The maximum field magnitude occurs when $\mathbf{M}$ is parallel to $\tilde{\mathbf{p}}$ and the minimum field magnitude occurs when $\mathbf{M}$ is perpendicular to $\tilde{\mathbf{p}}$. In the special case of $\hat{\boldsymbol{\Omega}}=\hat{\mathbf{p}}$, corresponding to the axial positions depicted in Fig. 1(a), then $\tilde{\mathbf{p}}=0$ and $|\mathbf{H}|_{\min }=|\mathbf{H}|_{\max }$.

The instantaneous angular velocity of the applied field as it rotates around $\hat{\omega}$ varies through each RPM cycle as well. If $\boldsymbol{\omega}$ and $\boldsymbol{\Omega}$ denote the instantaneous angular velocities of the local field and the RPM, respectively, then the relationship between $|\boldsymbol{\omega}|$ and $|\boldsymbol{\Omega}|$ can be found as

$$
|\boldsymbol{\omega}|=\left(\frac{|\mathbf{H}|_{\min }|\mathbf{H}|_{\max }}{|\mathbf{H}|^{2}}\right)|\boldsymbol{\Omega}| .
$$

Because $|\mathbf{H}|>0$ at all times, it is clear that the maximum angular velocity of the applied field occurs at the instant when the magnetic field strength $|\mathbf{H}|$ is the weakest.

With the field magnitude varying from $|\mathbf{H}|_{\min }$ to $|\mathbf{H}|_{\max }$, the maximum magnetic torque (and consequently the step-out frequency $|\boldsymbol{\omega}|_{\text {so }}$ ) varies from $\mu_{0}|\mathbf{m}||\mathbf{H}|_{\text {min }}$ to $\mu_{0}|\mathbf{m}||\mathbf{H}|_{\max }$ through each RPM revolution. Although the field magnitude cannot be controlled explicitly, $|\mathbf{p}|$ can be adjusted by moving the RPM close enough to the MAT, without changing the solution for $\hat{\omega}$, such that $|\mathbf{H}|_{\min }$, and thus the minimum step-out frequency, are sufficient for the desired application. It is also theoretically possible to adjust $|\mathbf{p}|$ dynamically throughout each RPM cycle such that $|\mathbf{H}|$ remains constant even when $\mathbf{p}$ is not in an axial position. Because rotating the field faster than $|\boldsymbol{\omega}|_{\text {so }}$ causes the MAT to become unsynchronized with the field, $|\boldsymbol{\Omega}|$ should be limited so that $|\boldsymbol{\omega}| \leq|\boldsymbol{\omega}|_{\text {so }}$ at all times.

\section{EXPERIMENTAL RESUlTS \& DiscusSiON}

The RPM is positioned with a Yaskawa Motoman MH5 6-DOF robotic manipulator (Fig. 2(a)), and consists of a cylindrical $25.4 \mathrm{~mm}$ diameter, $25.4 \mathrm{~mm}$ long, Grade-N42, diametrically magnetized (i.e., along the diameter) $\mathrm{NdFeB}$ permanent magnet (Fig. 2(b)) driven by a Maxon $24 \mathrm{~V}$ AMax DC motor with an Advanced Motion Controls servo control drive and amplifier. We demonstrate control of rotating MATs by actuating a rolling spherical device (Fig. 2(c)) and a threaded capsule-shaped device (Fig. 2(d)) similar to the design in [11], that produces translational motion along

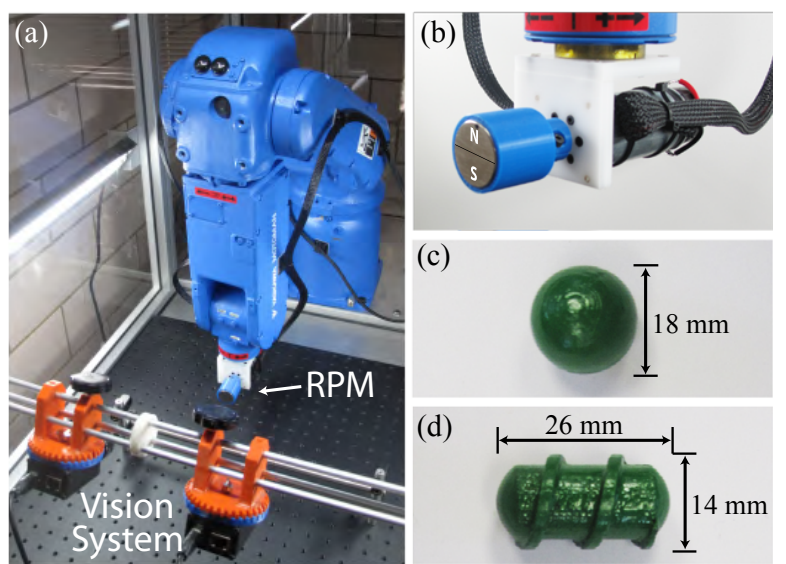

Fig. 2. The Yaskawa Motoman MH5 6-DOF robotic manipulator (a) is used to position the RPM (b) for point-dipole calibration and for actuating a spherical, rolling MAT (c) and a threaded screw MAT (d). The thread of the screw MAT has a pitch of $7 \mathrm{~mm}$ and is $1.5 \mathrm{~mm}$ deep. Both devices contain a $6.35 \mathrm{~mm}$ cube Grade-N52 NdFeB magnet.

its principal axis from rotation around the same axis. Both MATs contain a $6.35 \mathrm{~mm}$ cubic Grade-N52 NdFeB magnet positioned at the device's center-of-gravity, with the dipole moment oriented perpendicular to the device principal axis in the case of the threaded capsule. The MATs were constrained during experimentation in a clear PVC lumen with $22.2 \mathrm{~mm}$ inner diameter, lightly lubricated with personal lubricant jelly. The MAT position was obtained using a stereo vision system (Fig. 2(a)) consisting of two Basler A602FC cameras each fitted with a $4.5 \mathrm{~mm}$ fixed-focal-length lens producing sub-millimeter tracking accuracy at 30 frames-per-second.

The theory presented in Sec. II and Sec. III assumes that the RPM's magnetic field can be accurately predicted with the point-dipole model (1). The point-dipole model exactly predicts the field produced by a spherical permanent magnet and is an approximation for every other geometry that becomes accurate in the far field region. The magnetic flux density $\mathbf{B}$ of the RPM, where $\mathbf{B}=\mu_{0} \mathbf{H}$, was measured using a custom-made three-axis sensor. The sensor is constructed using three pairs of Allegro A1301 linear Hall-effect sensors mounted on the six sides of a $7 \mathrm{~mm}$ cube. Each sensor has a sensitivity of $25 \mathrm{~V} / \mathrm{T}$ and a range of $\pm 0.1 \mathrm{~T}$. The average measured value of the pair of sensors on two opposing faces approximates the component of the magnetic field at the cube's center in the normal direction to the faces. The magnitude of the RPM's dipole moment was found using a least-squares fit of the point-dipole model to 100 samples of the flux density measured per millimeter between $30 \mathrm{~mm}$ and $140 \mathrm{~mm}$ from the RPM center, resulting in $|\mathbf{M}|=12.7 \mathrm{~A} \cdot \mathrm{m}^{2}$ with $R^{2}=0.999$. The data and the fit are shown in Fig. 3 .

The accuracy of the fit and the correctness of the theory presented in Sec. II was verified by measuring the magnetic field while rotating the RPM at two arbitrary positions located $100 \mathrm{~mm}$ from the RPM center. The positions $\mathbf{p}_{1}=$ $[0,100,0]^{T} \mathrm{~mm}$ (Fig. 4(a)) and $\mathbf{p}_{2}=[81.61,57.79,0]^{T} \mathrm{~mm}$ (Fig. 4(b)) are described in the stationary coordinate system depicted at the top of Fig. 4. Figs. 4(c) and 4(d) show the 


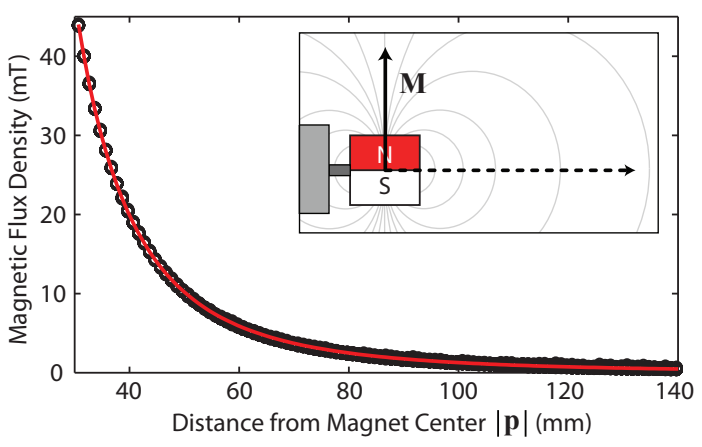

Fig. 3. The point-dipole model was fit to the measured magnetic flux density of the RPM at increasing distance from the magnet center resulting in $|\mathbf{M}|=12.7 \mathrm{~A} \cdot \mathrm{m}^{2}$ with $R^{2}=0.999 .100$ samples were taken per millimeter between $30 \mathrm{~mm}$ and $140 \mathrm{~mm}$ from the magnet center.

measured and predicted magnetic fields and axes of rotation at both positions projected onto the $\mathbf{x}-\mathbf{z}$ and $\mathbf{y}-\mathbf{z}$ planes. The RPM was rotated at a constant angular velocity of $1 \mathrm{rad} / \mathrm{s}$ in both positions. The predicted $\hat{\omega}$, obtained by (4), differed from the measured $\hat{\boldsymbol{\omega}}$, obtained using principal-component analysis (the direction of least variance), by $2.94^{\circ}$ for $\mathbf{p}_{1}$ and $0.73^{\circ}$ for $\mathbf{p}_{2}$. Fig. 4 clearly shows that the RPM magnetic field and its axis of rotation is closely predicted by the pointdipole model with $|\mathbf{M}|=12.7 \mathrm{~A} \cdot \mathrm{m}^{2}$.

To date, rotating MATs have been actuated in axial or radial positions where the applied field rotation is easy to visualize and the coupling between the RPM and the MAT is easy to understand. In the radial position, for example, the magnetic field rotates around an axis parallel to that of the RPM, although in the opposite direction. Actuation of the threaded MAT in the radial position is demonstrated in Fig. 5(a). While the MAT travels along the lumen (from right to left), the RPM position is maintained at $\mathbf{p}=[0,0,-125]^{T} \mathrm{~mm}$ (in the coordinate system depicted in Fig. 5) using the stereo vision system. Because the external thread converts rotation about the capsule's principal axis to propulsion parallel to its principal axis, it is intuitive that $\hat{\Omega}$ must be parallel to the $\mathbf{y}$ axis to make the threaded MAT travel along the lumen. In Fig. 5(a), the MAT travels at an average speed of $3.4 \mathrm{~mm} / \mathrm{s}$. Rotating MATs actuated in the radial position can be found in [5]-[9].

The theory presented in Sec. II enables operation of MATs in nonintuitive ways. Fig. 5(b) shows the threaded MAT actuated with the RPM position regulated to maintain $\mathbf{p}=$ $[0,-72.2,-102]^{T} \mathrm{~mm}$. After substituting the appropriate $\mathbf{p}$ and $\hat{\omega}=[0,-1,0]^{T}$ into (3), it can be found that the RPM rotation axis must be $\hat{\Omega}=[0,0,1]^{T}$. The RPM is rotated at $2.5 \mathrm{rad} / \mathrm{s}$ and causes the MAT to travel from right to left with an average speed of $1.5 \mathrm{~mm} / \mathrm{s}$ It is particularly nonintuitive that the necessary RPM rotation axis is perpendicular to the desired MAT rotation axis in this position. Note that the rotation axis of the applied field in an equivalent position is experimentally measured in Fig. 4(d).

Unlike uniform magnetic fields, nonuniform fields (such as those produced by the RPM) cause a magnetic force to be applied to the MAT. In Fig. 5(b), the magnetic force
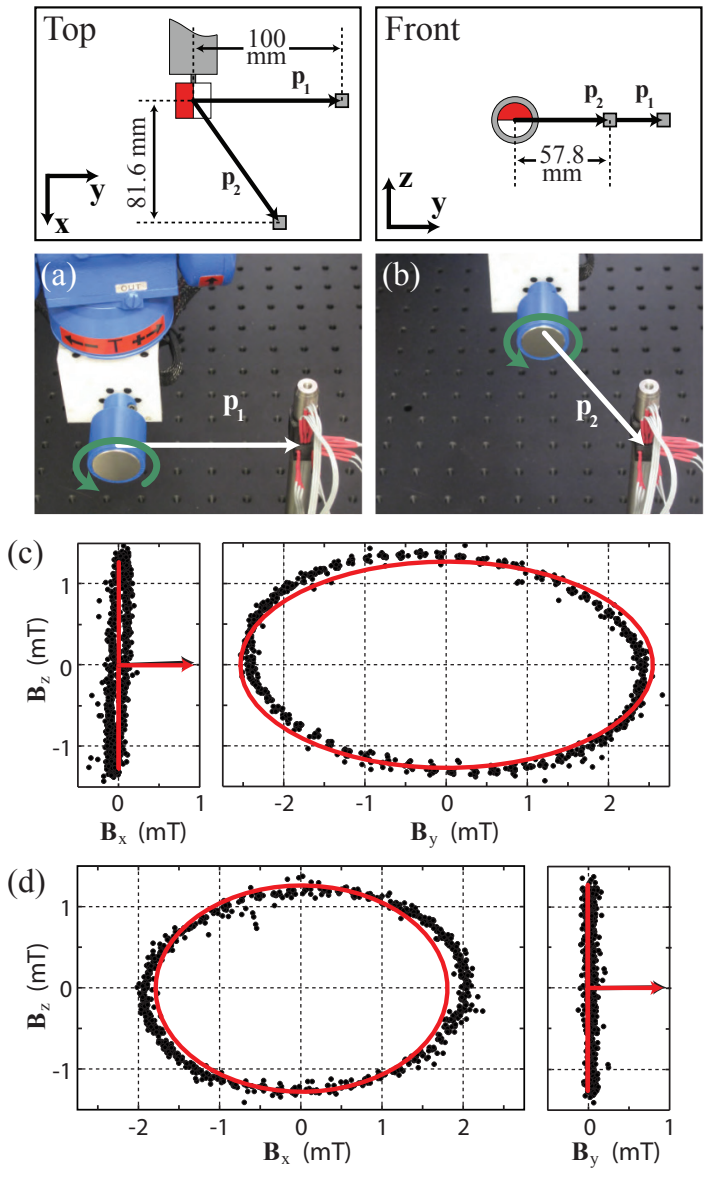

Fig. 4. The components of the magnetic flux density $\mathbf{B}=\mu_{0} \mathbf{H}$ measured and predicted at the positions $\mathbf{p}_{1}=[0,100,0]^{T} \mathrm{~mm}$ (a) and $\mathbf{p}_{2}=[81.61,57.79,0]^{T} \mathrm{~mm}(\mathrm{~b})$ are shown in (c) and (d), respectively. The predicted $\hat{\omega}$ at $\mathbf{p}_{1}$ and $\mathbf{p}_{2}$ differed from the measured $\hat{\omega}$ by $2.94^{\circ}$ and $0.73^{\circ}$, respectively. The custom field sensor used to obtain the data appears in the lower right corners of (a) and (b).

attracts the MAT in the direction of the RPM and against the desired motion. The ability to drive the MAT away from the RPM, albeit slowly, is likely to be an important advancement toward the control of in vivo devices. Stably driving a MAT away from a single permanent magnet, when solely applying magnetic force for actuation, would be challenging because the magnetic torque tends to align the MAT in configurations where the magnetic force is always attractive.

For medical applications, a magnetic force too large in magnitude can potentially cause trauma. Strategies have been developed to manage attractive magnetic force in the axial [8] and radial positions [7], though a method generalized to any position is the subject of future work. Although the attractive magnetic force must always be managed, it is not necessarily detrimental. Fig. 5(c) shows the MAT actuated with $\mathbf{p}=$ $[0,72.2,-102]^{T} \mathrm{~mm}$. In this position, a component of the magnetic force attracts the MAT in the desired direction of motion, producing an average speed of $4.3 \mathrm{~mm} / \mathrm{s}$, which is faster than the average speed in the radial position (Fig. 5(a)), where the force neither hinders nor helps actuation, and the position where the RPM trails the MAT (Fig.5(b)), 
(a)

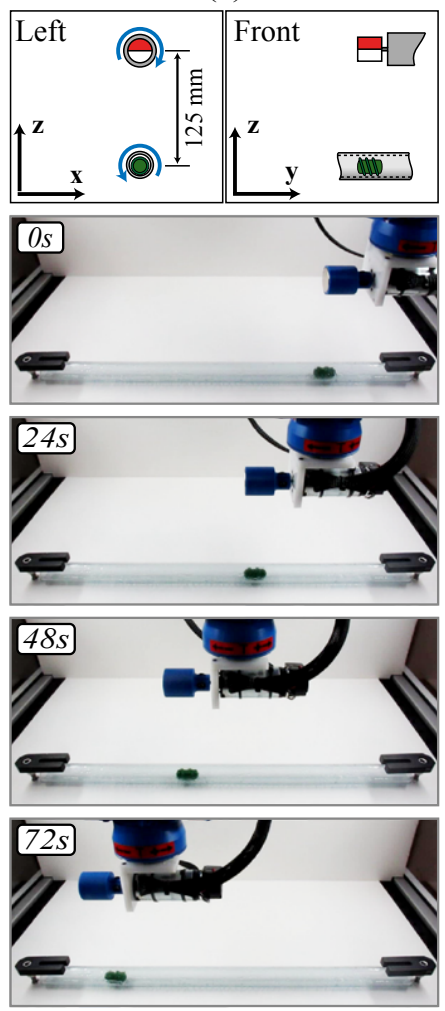

(b)
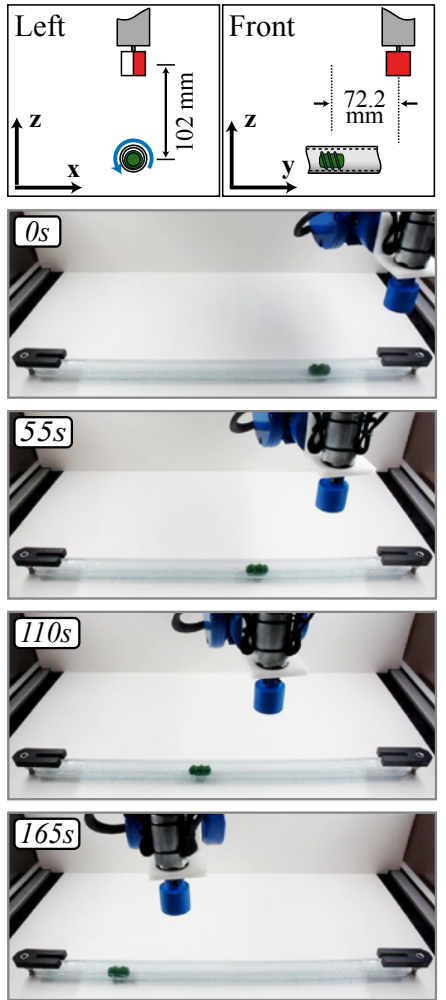

(c)
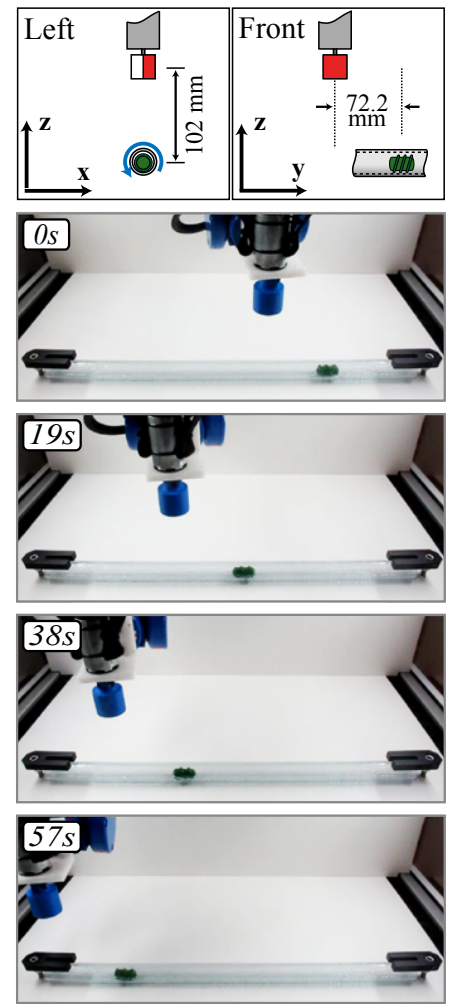

Fig. 5. The threaded capsule MAT is actuated in the (a) radial position with the $\mathrm{RPM}$ position regulated to maintain $\mathbf{p}=[0,0,-125]^{T}$ mm (in the coordinate system depicted above the captured images) where the necessary RPM rotation axis is intuitive. Captured images taken during operation with (b) the RPM position maintained at $\mathbf{p}=[0,-72.2,-102]^{T} \mathrm{~mm}$ and (c) at $\mathbf{p}=[0,72.2,-102]^{T}$ mm demonstrate the MAT being actuated where the necessary RPM rotation axis (found by applying (3)) is nonintuitive. In all three examples, $|\mathbf{p}|=125 \mathrm{~mm}$ and the RPM was rotated at a constant speed of $|\boldsymbol{\Omega}|=5 \mathrm{rad} / \mathrm{s}$. The scale drawings above the captured images depict the scene from direct left and front views.

where the force hinders actuation. This demonstrates that a rotating MAT can be simultaneously pulled, using the available magnetic force, and driven, using the available magnetic torque, resulting in faster MAT actuation.

In Fig. 5, the position of the RPM is regulated to maintain a constant relative position with respect to the MAT. Due to the presence of obstacles in a clinical setting that an RPM must avoid, it is unlikely that a MAT will always be actuated in this manner. The theory presented in Sec. II enables a MAT to be actuated in any position, which may change as the MAT or the RPM move in space, provided that the RPM rotation axis $\hat{\Omega}$ is adjusted according to (3). This is demonstrated in Fig. 6 which shows the spherical MAT being actuated while the RPM position (a) remains stationary, and (b) follows a trajectory independent of the MAT. In both examples, the RPM position follows the predifined position trajectory (remaining stationary in the case of (a)) while the RPM's instantaneous rotation axis $\hat{\Omega}$ is set according to (3) using the known RPM position and the instantaneous MAT position, obtained from the stereo vision system, to update the instantaneous position vector $\mathbf{p}$. In both examples, $\hat{\boldsymbol{\omega}}$ is maintained at $\hat{\boldsymbol{\omega}}=[-1,0,0]^{T}$ (in the same coordinate system as Fig. 5), and the RPM is rotated at $|\Omega|=0.25 \mathrm{rad} / \mathrm{s}$.

Fig. 6(a) shows superimposed still images demonstrating the MAT being rolled from left to right while the RPM position remains stationary. In the case of Fig. 6(b), the MAT rolls from left to right while the RPM follows a rectangular step trajectory, where the RPM initially travels parallel to the lumen at $3.16 \mathrm{~mm} / \mathrm{s}$ until it jogs vertically at $t=33 \mathrm{~s}$ with a rate of $9.47 \mathrm{~mm} / \mathrm{s}$. At $t=44 \mathrm{~s}$, the RPM continues parallel to the lumen until $t=77 \mathrm{~s}$, when the RPM moves back to its original height at $9.47 \mathrm{~mm} / \mathrm{s}$. The RPM then finishes moving parallel to the lumen at $3.16 \mathrm{~mm} / \mathrm{s}$. It is easy to imagine this trajectory being used to avoid an obstacle. In both examples, the MAT is always in stable control and the RPM is never in an axial or radial position. Although both RPM trajectories are arbitrary, they demonstrate MAT actuation that was not possible using prior methods, which limit the RPM to axial or radial positions. In practice, RPM position trajectories should be planned to prevent loss of control authority, take advantage of available magnetic force, and avoid obstacles.

The RPM's magnetic field scales homothetically, meaning that the field of an RPM, whose dimensions have been scaled by a factor $s$, measured at the position $s \mathbf{p}$, is the same as that of an unscaled RPM measured at the position $\mathbf{p}$. This can be verified using (1). Because the dipole magnetization $|\mathbf{M}|$ is proportional to the RPM's volume, scaling by $s$ causes the magnetic dipole to become $s^{3}|\mathbf{M}|$. Operating the MAT at an equivalently scaled distance away makes $\mathbf{p}$ become $s \mathbf{p}$. After substituting $s^{3}|\mathbf{M}|$ and $s \mathbf{p}$ into (1), the factor $s^{3}$ appears 

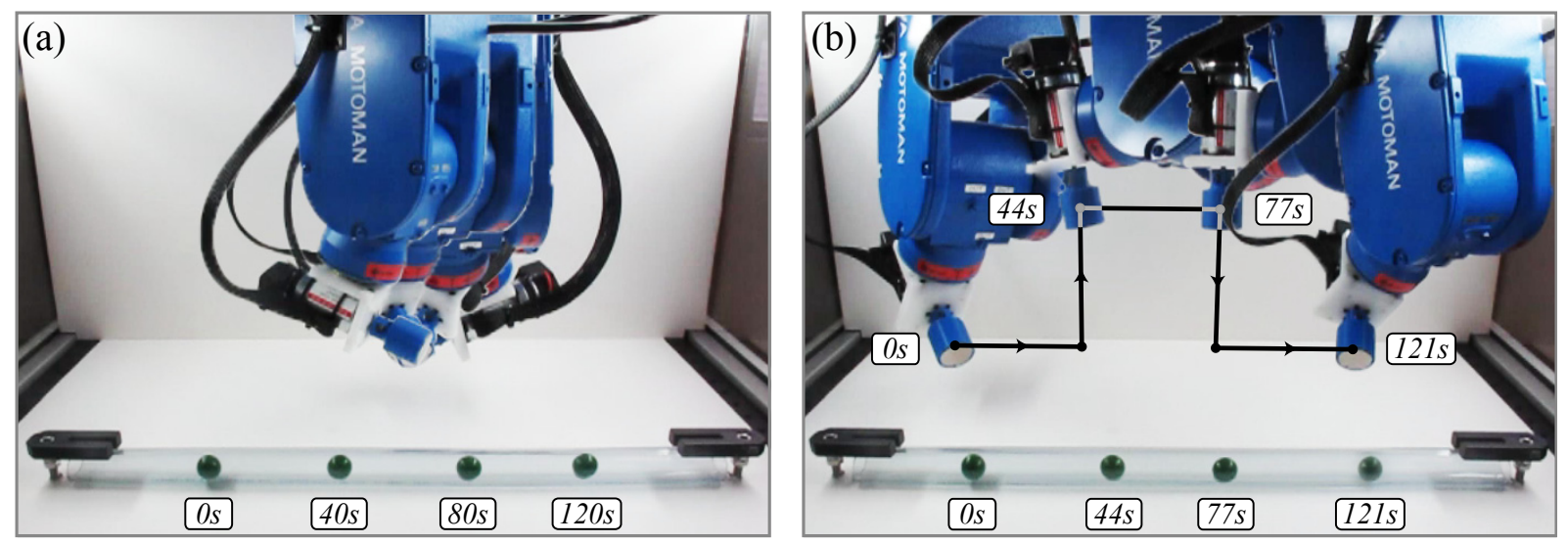

Fig. 6. The spherical MAT (Fig. 2(c)) is actuated with the RPM position remaining stationary (a) and following a rectangular step trajectory (b). In both examples, the RPM position follows the trajectory (or remains stationary) in an open-loop fashion, independent of the MAT position. The orientation of the RPM's rotation axis $\hat{\boldsymbol{\Omega}}$ is constantly updated to maintain $\hat{\boldsymbol{\omega}}=[-1,0,0]^{T}$ (in the same coordinate system as Fig. 5) by applying (3), using the known RPM position and the instantaneous MAT position (obtained from the vision system) to update the relative position $\mathbf{p}$. $|\boldsymbol{\Omega}|=0.25 \mathrm{rad} / \mathrm{s}$ in both cases.

in both the numerator and the denominator and can be cancelled, making the field of the scaled RPM at equivalently scaled distances the same as without scaling. It can be shown that the magnetic force $\mathbf{F}=\mu_{0}(\mathbf{m} \cdot \nabla) \mathbf{H}$ is amplified by the scalar $3 \mu_{0}|\mathbf{M}| / 4 \pi|\mathbf{p}|^{4}$ [15]. Substituting $s^{3}|\mathbf{M}|$ and $s \mathbf{p}$ into this scalar shows that the magnetic force scales as $s^{-1}$. This implies that when the RPM dimensions are scaled by $s$ and the MAT is operated at an equivalently scaled distance, the magnetic field (and thus the magnetic torque) remains the same while the magnetic force is diminished. This demonstrates that the magnetic fields generated by the RPM will scale well for clinical use, however, if the magnetic force is expected to assist actuation (as demonstrated in Fig. 5(c)), then diminishing the force may be undesireable. Fully understanding the magnetic force and how it should best be applied or managed is the subject of future work.

\section{CONCLUSION}

It has been shown that when a magnetic dipole, such as a permanent magnet, is rotated around a fixed axis such that the dipole is perpendicular to the axis of rotation, the magnetic field vector at every point in space also rotates around a fixed axis. We have reformulated and applied this property to actuate untethered, rotating magnetic devices confined to a lumen. To date, rotating MATs operated using a single RPM have been actuated in positions where the magnetic field behavior is simple and easy to visualize. Constraining the RPM to these positions may preclude the use of RPM actuation in clinical settings due to the presence of obstacles. The method presented herein, however, allows a rotating MAT to be operated in any position, which we experimentally demonstrate by driving a spherical rolling MAT and a threaded capsule MAT with the RPM positioned arbitrarily in space but with the RPM orientation determined in a closed-loop fashion by the MAT position. Eliminating the need for MATs to be constrained to simple positions is a significant step toward making rotating MATs actuated by a single RPM clinically feasible.

\section{REFERENCES}

[1] B. J. Nelson, I. K. Kaliakatsos, and J. J. Abbott, "Microrobots for minimally invasive medicine," Апnи. Rev. Biomed. Eng., vol. 12, pp. $55-85,2010$.

[2] J. L. Toennies, G. Tortora, M. Simi, P. Valdastri, and R. J. Webster III, "Swallowable medical devices for diagnosis and surgery: the state of the art," J. Mech. Eng. Sci., vol. 224, no. 7, pp. 1397-1414, 2010.

[3] G. Ciuti, P. Valdastri, A. Menciassi, and P. Dario, "Robotic magnetic steering and locomotion of capsule endoscope for diagnostic and surgical endoluminal procedures," Robotica, vol. 28, no. 2, pp. 199 207, 2010.

[4] M. P. Kummer, J. J. Abbott, B. E. Kratochvil, R. Borer, A. Sengul, and B. J. Nelson, "Octomag: An electromagnetic systems for 5-DOF wireless micromanipulation," IEEE Trans. Robot., vol. 26, no. 6, pp. 1006-1017, 2010

[5] M. T. Hou, H.-M. Shen, G.-L. Jiang, C.-N. Lu, I.-J. Hsu, and J. A. Yeh, "A rolling locomotion method for untethered magnetic microrobots," Appl. Phys. Lett., vol. 96, no. 024102, pp. 1-3, 2010.

[6] S. Yim and M. Sitti, "Design and rolling locomotion of a magnetically actuated soft capsule endoscope," IEEE Trans. Robot., vol. 28, no. 1, pp. 183-194, 2012.

[7] A. W. Mahoney and J. J. Abbott, "Managing magnetic force applied to a magnetic device by a rotating dipole field," Appl. Phys. Lett., vol. 99, no. 134103, pp. 1-3, 2011.

[8] T. W. R. Fountain, P. V. Kailat, and J. J. Abbott, "Wireless control of magnetic helical microrobots using a rotating-permanent-magnet manipulator," in Proc. IEEE Int. Conf. Robotics and Automation, 2010, pp. $576-581$.

[9] J.-S. Lee, B. Kim, and Y.-S. Hong, "A flexible chain-based screw propeller for capsule endoscopes," Int. J. of Prec. Eng. and Manuf., vol. 10, no. 4, pp. 27-34, 2009.

[10] A. Ghosh and P. Fischer, "Controlled propulsion of artificial magnetic nanostructured propellers," Nano Lett., vol. 9, no. 6, pp. 2243-2245, 2009.

[11] M. Sendoh, K. Ishiyama, and K. I. Arai, "Fabrication of magnetic actuator for use in a capsule endoscope," IEEE Trans. Magn., vol. 39, no. 5, pp. 3232-3234, 2003.

[12] L. Zhang, J. J. Abbott, L. X. Dong, B. E. Kratochvil, D. Bell, and B. J. Nelson, "Artificial bacterial flagella: Fabrication and magnetic control," Appl. Phys. Lett., vol. 94, no. 064107, pp. 1-3, 2009.

[13] K. Ishiyama, K. I. Arai, M. Sendoh, and A. Yamazaki, "Spiral-type micro-machine for medical applications," J. of Micromechatronics, vol. 2, no. 1, pp. 77-86, 2003.

[14] E. Paperno, I. Sasada, and E. Leonovich, "A new method for magnetic position and orientation tracking," IEEE Trans. Magn., vol. 37, no. 4, pp. 1938-1940, 2001.

[15] E. P. Furlani, Permanent magnet and electromechanical devices: materials, analysis, and applications, 1st ed. San Diego, California: Academic Press, 2001. 